\title{
Challenges during Metis-Solar Orbiter commissioning phase
}

\section{Marco Romoli, Vincenzo Andretta, Alessandro Bemporad, Marta Casti, Vania Da Deppo, et al.}

Marco Romoli, Vincenzo Andretta, Alessandro Bemporad, Marta Casti, Vania Da Deppo, Yara De Leo, Michele Fabi, Silvano Fineschi, Fabio Frassetto, Catia Grimani, Klaus Heerlein, Petr Heinzel, Giovanna Jerse, Federico Landini, Alessandro Liberatore, Enrico Magli, Giampiero Naletto, Gianalfredo Nicolini, Maurizio Pancrazzi, Maria Guglielmina Pelizzo, Paolo Romano, Clementina Sasso, Udo Schühle, Alessandra Slemer, Daniele Spadaro, Thomas Straus, Roberto Susino, Luca Teriaca, Michela Uslenghi, Cosimo Antonio Volpicelli, Paola Zupella, "Challenges during Metis-Solar Orbiter commissioning phase," Proc. SPIE 11852, International Conference on Space Optics - ICSO 2020, 118525A (11 June 2021); doi: 10.1117/12.2599944

SPIE Event: International Conference on Space Optics - ICSO 2021, 2021, Online Only 


\section{International Conference on Space Optics-ICSO 2020}

Virtual Conference

30 March-2 April 2021

Edited by Bruno Cugny, Zoran Sodnik, and Nikos Karafolas
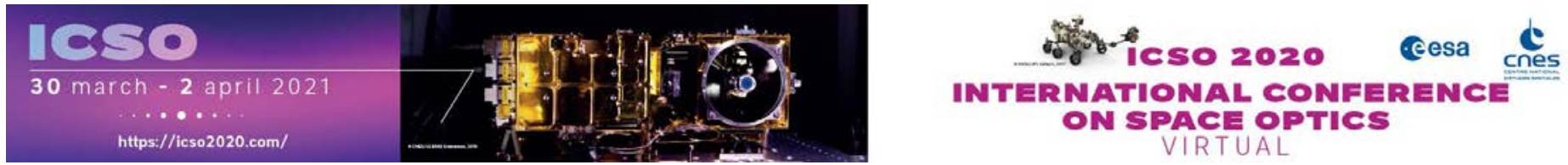

\section{Challenges during Metis-Solar Orbiter commissioning phase}

\section{Cesa isso procedings denes}




\title{
Challenges during Metis-Solar Orbiter commissioning phase
}

\author{
Marco Romoli ${ }^{\mathrm{a}, \mathrm{o}}$, Vincenzo Andretta ${ }^{\mathrm{b}}$, Alessandro Bemporad ${ }^{\mathrm{c}}$, Marta Casti $^{\mathrm{d}}$, Vania Da \\ Deppo $^{\mathrm{e}}$, Yara De Leo, ${ }^{\mathrm{f},}$, Michele Fabi ${ }^{\mathrm{g}}$, Silvano Fineschic ${ }^{\mathrm{c}}$, Fabio Frassetto ${ }^{\mathrm{e}}$, Catia Grimani ${ }^{\mathrm{g}}$, \\ Klaus Heerlein ${ }^{\mathrm{f}}$, Petr Heinzel ${ }^{\mathrm{h}}$, Giovanna Jerse ${ }^{\mathrm{i}}$, Federico Landinic ${ }^{\mathrm{c}}$, Alessandro Liberatore ${ }^{\mathrm{c}}$, \\ Enrico Maglij, Giampiero Naletto ${ }^{\mathrm{k}}$, Gianalfredo Nicolini ${ }^{\mathrm{c}}$, Maurizio Pancrazzi ${ }^{\mathrm{c}}$, Maria \\ Guglielmina Pelizzo ${ }^{\mathrm{e}}$, Paolo Romano ${ }^{1}$, Clementina Sasso ${ }^{\mathrm{b}}$, Udo Schühle ${ }^{\mathrm{f}}$, Alessandra Slemer ${ }^{\mathrm{e}}$, \\ Daniele Spadarol, Thomas Straus ${ }^{\mathrm{b}}$, Roberto Susino ${ }^{\mathrm{c}}$, Luca Teriaca ${ }^{\mathrm{f}}$, Michela Uslenghi ${ }^{\mathrm{m}}$, \\ Cosimo Antonio Volpicellic, and Paola Zuppella ${ }^{\mathrm{e}}$ \\ ${ }^{a}$ Università di Firenze, Italy \\ ${ }^{\mathrm{b}} \mathrm{INAF}$ - Osservatorio Astronomico di Capodimonte, Napoli, Italy \\ ${ }^{\mathrm{c}}$ INAF - Osservatorio Astrofisico di Torino, Italy \\ ${ }^{\mathrm{d}}$ Altec SpA, Torino, Italy \\ ${ }^{\mathrm{e}} \mathrm{CNR}$ - IFN, Padova, Italy \\ ${ }^{\mathrm{f}} \mathrm{MPS}$, Göttingen, Germany \\ "Università di Urbino "Carlo Bo" and INFN, Italy \\ ${ }^{\mathrm{h}}$ Academy of Science, Astronomical Institute, Ondrejov, Czech Republic \\ ${ }^{i}$ INAF - Osservatorio Astronomico di Trieste, Italy \\ ${ }^{\mathrm{j} P o l i t e c n i c o,}$ Torino, Italy \\ ${ }^{\mathrm{k}}$ Università di Padova, Italy \\ ${ }^{1}$ INAF - Osservatorio Astrofisico di Catania, Italy \\ mINAF - IASF, Milano, Italy \\ ${ }^{n}$ Università di Catania, Italy \\ ${ }^{\circ} \mathrm{INAF}$ - Osservatorio Astrofisico di Arcetri, Firenze, Italy
}

\begin{abstract}
Metis is the visible light and UV light imaging coronagraph on board the ESA-NASA mission Solar Orbiter that has been launched February 10th, 2020, from Cape Canaveral. Scope of the mission is to study the Sun up close, taking high-resolution images of the Sun's poles for the first time, and understanding the Sun-Earth connection. Metis coronagraph will image the solar corona in the linearly polarized broadband visible radiation and in the UV HI Ly- $\alpha$ line from 1.6 to 3 solar radii when at Solar Orbiter perihelion, providing a diagnostics, with unprecedented temporal coverage and spatial resolution, of the structures and dynamics of the full corona. Solar Orbiter commissioning phase big challenge was Covid-19 social distancing phase that affected the way commissioning of a spacecraft and its payload is typically done. Metis coronagraph on-board Solar Orbiter had its additional challenges: to wake up and check the performance of the optical, electrical and thermal subsystems, most of them unchecked since Metis delivery to spacecraft prime, Airbus, in May 2017. The roadmap to the fully commissioned coronagraph is here described throughout the steps from the software functional test, the switch on of the detectors of the two channels, UV and visible, to the optimization of the occulting system and the characterization of the instrumental stray light, one of the most challenging features in a coronagraph.
\end{abstract}

Keywords: Metis, Solar Orbiter, Coronagraph, Space Instrumentation

Further author information: (Send correspondence to M. Romoli)

M. Romoli: E-mail: marco.romoli@unifi.it, Telephone: +39 0552755233 


\section{INTRODUCTION}

Solar Orbiter ${ }^{1}$ was launched on February 10, 2020, from Cape Canaveral, and went through the Near Earth Commissioning Phase (NECP), that lasted till the June 12, 2020. During the NECP the spacecraft and the payload were functionally checked and their performance verified and, where possible, optimized. All these activities were performed using a direct link with the spacecraft, sending commands and receiving telemetry in quasi real time, due to the light travel time from the ground station to the spacecraft and back. During NECP, Metis coronagraph was switched on, functionally tested and characterized. In particular, the two mechanisms of the instrument, the one-shot sealing cap and the internal occulter positioning mechanism (IOM), were activated.

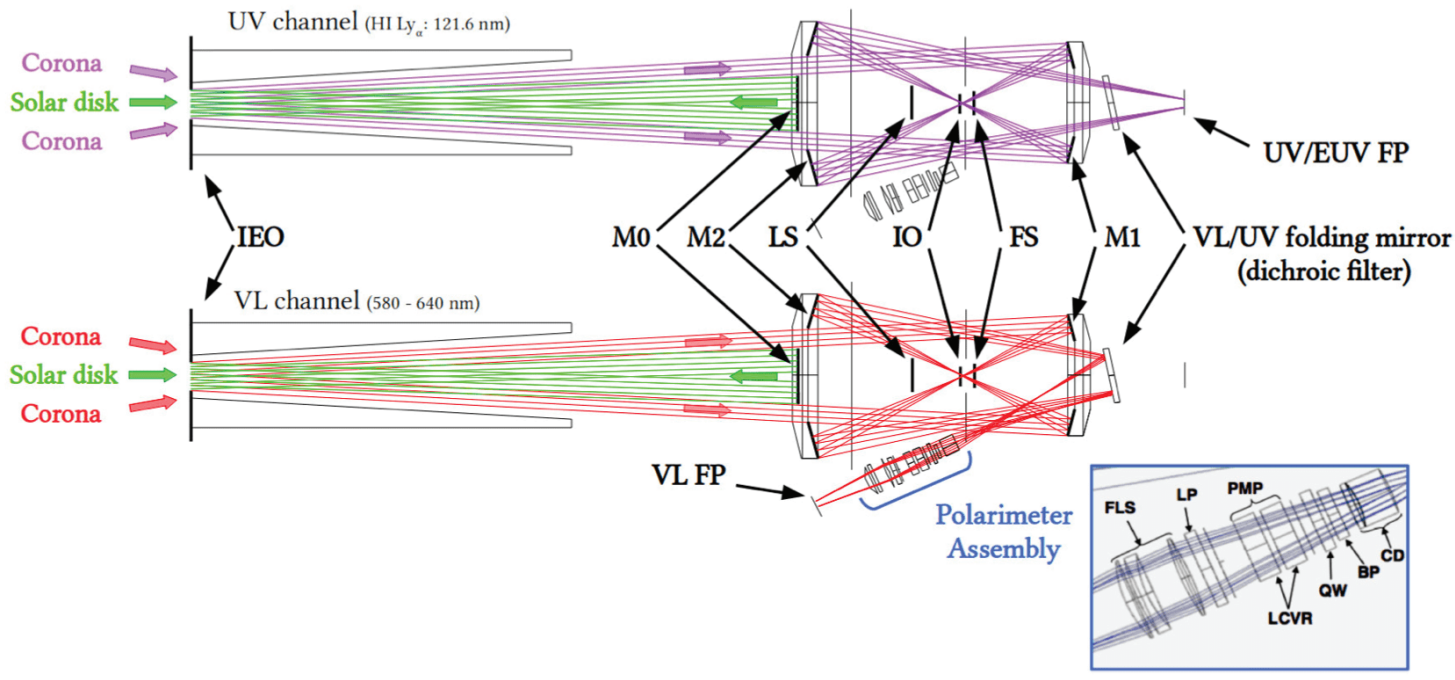

Figure 1. Metis ray trace for the UV and VL channels with a zoom on the VL polarimeter assembly.

Metis, ${ }^{2}$ the Solar Orbiter coronagraph, is designed to image the solar corona in an annular field of view centered on the Sun disk center and covering the range from 1.6 to 2.9 degrees. Metis is an externally occulted coronagraph with a novel inverted optical configuration ${ }^{3}$ (Fig.1) that takes images in the broadband linearly polarized visible light $(580-640 \mathrm{~nm})$ and, for the first time simultaneously over the entire field of view, in the narrowband $\mathrm{HI} \mathrm{Ly}-\alpha$ at $121.6 \mathrm{~nm}$. The instrument consists of a single on-axis gregorian telescope with the UV and the VL channel, that are separated by an interference filter tuned at $121.6 \mathrm{~nm}$ that reflects the visible light and transmits the UV light. NECP activity for Metis started on February 27 with the switch on and the short functional test to verify the operativeness of most of the instrument subsystems. The completion of Metis commissioning required 14 activity sessions that were completed on June 8, despite the covid-19 pandemic outbreak, with a few tests left and a bunch of features to be solved during the mission cruise phase. Nevertheless, these features do not block Metis science observations and the NECP was considered successfully completed.

\section{THE IMPACT OF COVID-19 PANDEMIC ON METIS OPERATIONS}

NECP for Metis was supposed to be carried on at the Solar Orbiter mission control center (MOC) at ESOC in Darmstadt, where the instrument team needed many representatives on site. Two or three from the team were allowed in a dedicated Solar Orbiter control room while the other representatives worked from a dedicated support area. Metis operations team would have been supported by a dedicated MOC engineer to coordinate the real time activity and the management of unexpected behaviors or anomalies to be solved within the session or reprogrammed. The presence at ESOC has been possible only for the first two activities because the outbreak of the Covid-19 pandemic brought to a sudden stop the possibility to travel to ESOC. The Italian-German-Czech team behind the Metis coronograph was just getting ready to switch on the instrument for the first time, on 
February 27, when the decision was made that people from coronavirus hotspots in Italian regions, Piemonte and Lombardia, were no longer allowed to enter ESOC for safety reasons. For the second activity on March 6 only two representatives from a considered safe region of Italy were allowed in. Afterwords, a strict lockdown in both Germany and Italy forced to perform all the activities from home. The activity leaders were connected on one side via gmeet with the rest of the Metis team and via webex with MOC. The situation became even more serious when several workers at ESOC tested positive for the virus, and the site effectively closed. The instruments were switched off on March 22 and NECP went on hold and it was not clear when the instruments could come back online. NECP was resumed about a week later. A skeleton staff returned and, with full social distancing measures in place, began working remotely with the instrument teams to get the commissioning done. Security concerns required ingenious solutions such as viewing the ESOC control screens, which showed the data from the spacecraft, via a webcam. The main drawback of the 'home-office' set-up was the added delay between the action being performed by the spacecraft and the data about the status of the operation reaching the team. In addition to that, it was difficult to keep the instrument team in contact from home and running the link with ESOC without voice interferences. The NECP ended with the first "virtual" ESA Mission Commissioning Results Review (MCRR) that declared NECP successful.

\section{METIS COMMISSIONING ACTIVITIES}

The Metis commissioning phase was planned to take place entirely in the NECP. The instrument was verified and tested on-ground during two short periods in December 2016 and in April 2017. In order to maintain the demanding cleanliness requirements during the on-ground instrument testing, except when in vacuum, during the integration on the spacecraft and the full spacecraft activity till shortly before launch, Metis instrument was sealed by a protection cap. A Nitrogen purging flow was continuously provided to gently overpressurize the telescope cavity preventing the entrance of contaminants. After launch, the outgassing phase lasted about one month, a period during which the contamination/protection doors were held closed. An additional fast outgassing time (5 days) with the heat shield door closed was performed after the cap ejection. The commissioning phase aims at verifying the electrical, mechanical, optical, thermal, and software functionalities of the instrument. Details on the instrument operations and on the commissioning activities can be found in the technical documents: Metis User Manual (METIS-ATI-MA-001) and the Metis Commissioning report (METIS-UNIFI-RPT-041 (available upon request). Table 1 lists all Metis NECP activities with the main objective of the session. Each activity was performed in a few hours. All activities were successful with the exception of those highlighted in italics in the table.

Table 1. Metis commissioning activity as-run.

\begin{tabular}{|l|c|l|}
\hline \multicolumn{1}{|c|}{ Activity } & Date & Activity description \\
\hline IT-1 & $27 / 02 / 2020$ & Short functional test (SFT) \\
\hline IT-2 & $06 / 03 / 2020$ & VL and UV dark images, Annealing heater test \\
\hline IT-3 & $13 / 03 / 2020$ & Cap ejection: VL first light \\
\hline IT-4\#1 & $06 / 04 / 2020$ & UV offset map upload \\
\hline IT-4\#2 & $08 / 04 / 2020$ & UVD High voltage \\
\hline IT-4\#3 & $23 / 04 / 2020$ & UV first light and darks \\
\hline IT-6A & $11 / 05 / 2020$ & Stray light check with roll \\
\hline IT-6B1 & $15 / 05 / 2020$ & Internal Occulter coarse alignment \\
\hline RSC & $28 / 05 / 2020$ & Coordinated campaign: Remote Sensing Co-alignment \\
\hline IT-5 & $29 / 05 / 2020$ & Cosmic Ray algorithm, compression and radialization test \\
\hline IIC & $29 / 05 / 2020$ & Coordinated campaign: Inter-Instrument Communication \\
\hline EMC & $02 / 06 / 2020$ & Coordinated campaign: Electromagnetic Cleanliness \\
\hline IT-6B2 & $03 / 06 / 2020$ & Internal Occulter fine tuning \\
\hline IT-7 & $08 / 06 / 2020$ & Off-pointing stray light characterization and Polarimeter test \\
\hline
\end{tabular}


Throughout the full commissioning the thermal behaviour of the instrument was verified against the thermal model.

Hereafter, the main achievements of commissioning are described in more detail.

\subsection{Detectors Calibration Frames}

The Metis detection systems consists of the VL detector (VLD) and the UV detector (UVD). The VLD is a CMOS-APS sensor with 2048 by 2048 pixels of $10-\mu \mathrm{m}$ size. The UVD is an intensified CMOS-APS camera, consisting of a microchannel plate (MCP) coupled to the APS (1024 by $102415-\mu \mathrm{m}$ pixels) via a fibre-optic taper and a phosphorus screen. ${ }^{2,4,5}$ The UVD has two operative modes: Analog Mode and Photon Counting Mode (PCM). The Analog Mode requires a moderate High Voltage applied to the MCP and it is the baseline UV acquisition scheme. The PCM will be used when the rate of the impinging photons will be very low or in case of a detector reduced quantum efficiency late in the mission; it requires the acquisition and the subtraction of an offset map on board. The failure of the offset map acquisition during NECP together with a not fully tested PCM on ground has lead to the decision of not commissioning the PCM, and debug the mode on a reference model on ground. A new version of the software with this and other bug fixes is foreseen to be uploaded to the spacecraft before the end of the cruise phase. The VLD operations are designed to perform the linear polarization measurement of the solar corona brightness. For this scope the VLD operates together with the polarimeter, described in section 3.5, acquiring 4 images with different polarization angles. A demodulation matrix, applied to the images, will return the Stokes vector for the linear polarization. Both detector are operated averaging on board a NDIT number of individual images exposed for a given Detector Integration Time (DIT). The UV detector has two steps of NDITs: NDIT1 is the number of exposures averaged in hardware mode, used only when the frame rate is too high to process the images by software, although the HW algorithm introduces a small approximation, while NDIT2 is the same of the NDIT for the VLD. By "cadence" is meant the time interval between two subsequent acquired images. One of the first activities performed when the cap was still sealing the instrument is the characterization of the dark images with both detectors. The detectors temperature is stabilized within 2 degrees range by means of external radiators and heaters (survival heaters) mounted on the Metis thermal interfaces with the spacecraft. The operative ranges at the interfaces are $\left[-32^{\circ} \mathrm{C} ;-30^{\circ} \mathrm{C}\right]$ for the $\mathrm{UV}$ detector (Medium Element) and $\left[-42^{\circ} \mathrm{C},-40^{\circ} \mathrm{C}\right]$ for the VL detector (Cold Element). Inside these ranges the dark signal will remain constant. VL and UV images are also corrected for the flat field flat field response (dominated, in the UVD, by the micro-channel plate). Flat fields have been measured on ground on the detector sub-systems.

\subsubsection{VL Dark and Bias}

After the ejection of the Metis cap it is not anymore possible to acquire further dark images. In fact, the heat shield door is not light tight and the scattering inside the instrument is large enough to saturate the sensor in a couple of seconds.

The dark map obtained during IT-2 (see Fig.2) will be used throughout the mission, with appropriate rescaling when necessary. The dark signal was acquired using increasing DITs. Fig.3 shows the average dark current computed in a 512x512 pixels central box and their linear fit. The fit parameters and their errors are also reported in figure.

The bias map and the dark map were extracted as, respectively, the constant parameter and the angular coefficient of the linear fit, pixel by pixel. The bias map is shown in Fig.4. As expected, the main component of the fixed pattern is given by a column pattern, being associated to the column amplifiers. There is also an even-odd rows effect, due to the fact that even and odd rows are readout by different column amplifiers.

Bias after cap ejection, since no dark images are available, are computed with the same approach using images taken at two or more different DITs and extrapolated to DIT $=0$ s. 


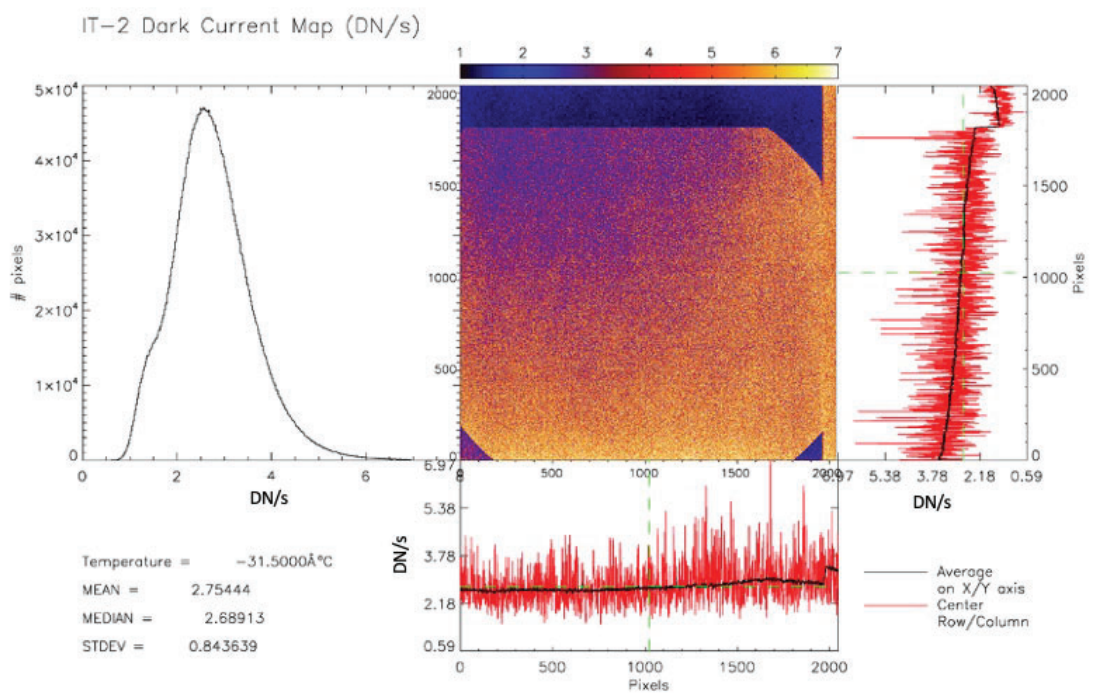

Figure 2. VL dark map obtained during IT-2 (center). The dark histogram (left). Vertical cut through the image (right and bottom).

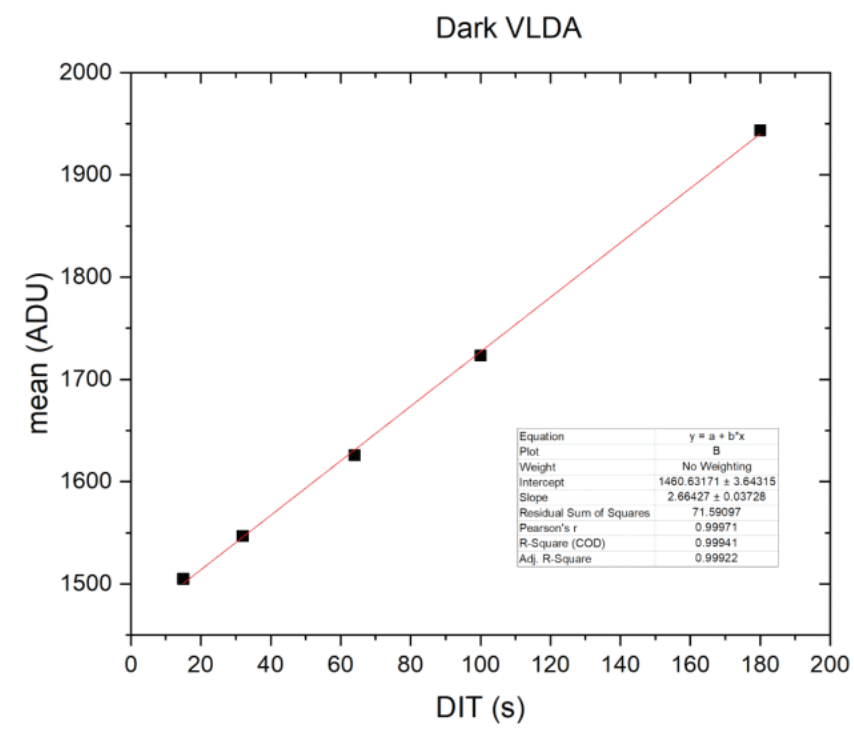

Figure 3. VL average dark linearity $\left(T_{\text {sensor }}=-31.5^{\circ} \mathrm{C}, \sigma_{T}=0.5^{\circ} \mathrm{C}\right)$.

\subsubsection{UV Dark and Bias}

Images specifically devoted to the characterization of the dark of the UVDA have been acquired with High Voltages off during IT-2 and IT-4.3. The dark signal has been measured at different exposure times, and at different parameters NDIT1/NDIT2. The stability in time has also been checked, and a transient effect at the beginning of each acquisition, already noticed in the on-ground calibration of the instrument, has been studied. As tested during the ground calibration, the dark signal is independent on the voltage applied to the screen gap (this is crucial in order to be able to use dark frames acquired with the HV switched off, thus providing the 


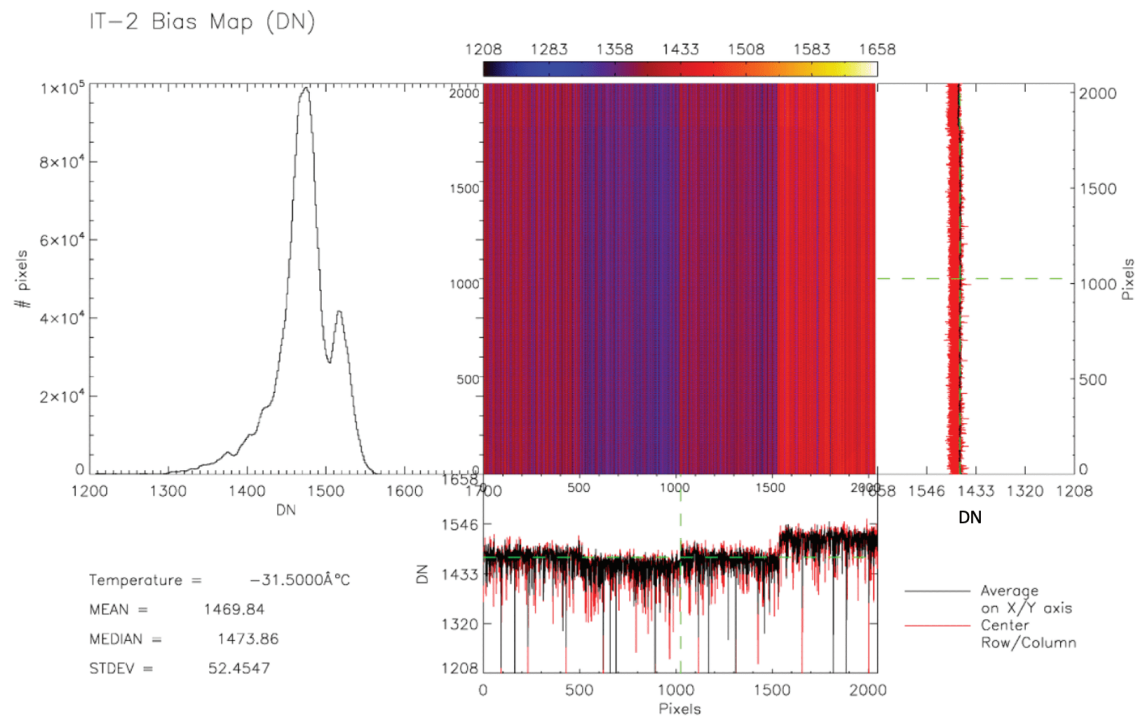

Figure 4. VL bias from IT-2.

possibility of acquiring dark frames even in presence of light).

Dark frames are being acquired during nominal acquisitions as part of the in-flight calibration activities that are scheduled for each observing window (see Fig.5). Additional investigation on the behaviour of the UVD is still on going, because of an anomaly encountered in the UVD images, where the signal depends on the choice of the parameters NDIT1, and NDIT2.

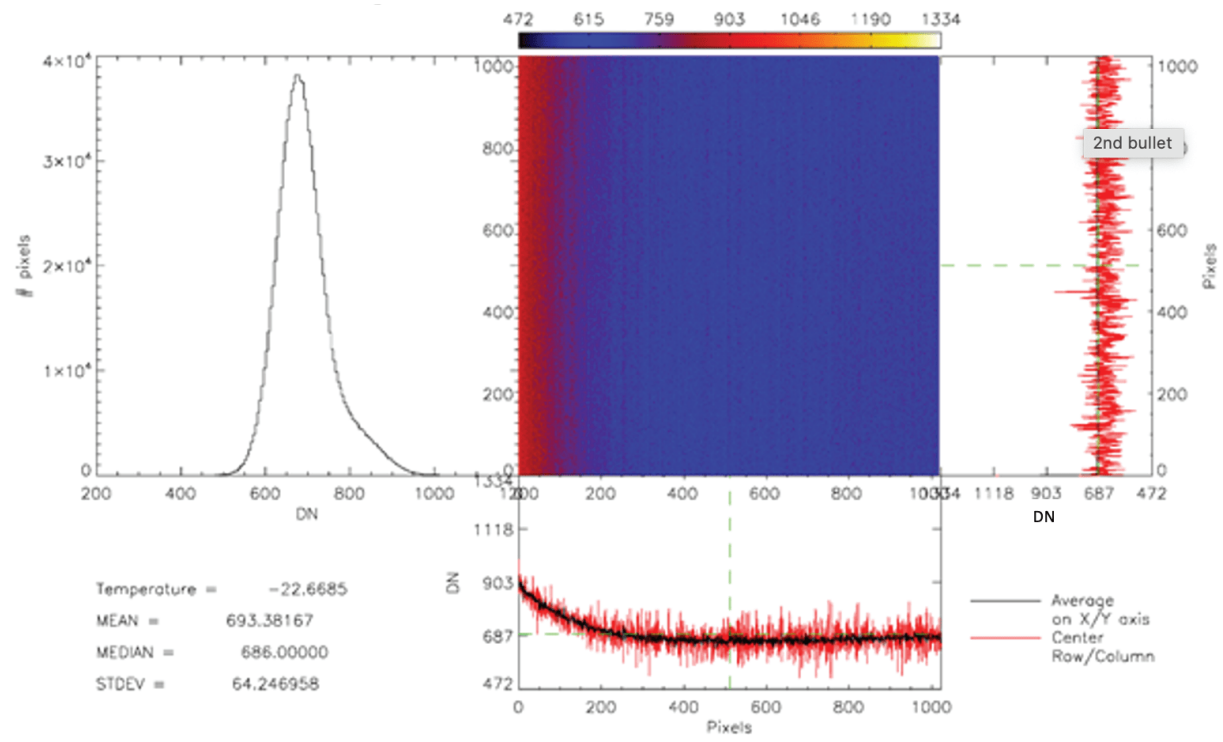

Figure 5. UV dark+bias map obtained during IT-2 (center) with DIT $=1 \mathrm{~s}$, NDIT1=4, and NDIT2=8. The dark histogram (left). Vertical cut through the image (right and bottom).

The dark signal vs. DIT is shown in Fig.6 in comparison of a similar test performed on-ground in a comparable 
temperature range. Non-linearity is clearly apparent, most likely due to intrinsic non-linearity in the dark + bias response of the sensor. Already for this reason, dark frames matching the adopted exposure time should be always acquired.

A temporal evolution of the transient was observed during ground testing at instrument level and has been studied with targeted observations in the IT-4.3 commissioning activity. Each time the sensor integration time is changed, the level of the signal slowly changes in the subsequent frames until stabilization after several tens of seconds. The temporal evolution of each series (with different cadences) is in excellent agreement with the others, which indicates that the transient is characterized by a reproducible temporal evolution, well modeled by a sigmoidal function. The signal stabilizes after $\sim 80-100 s$. This is crucial in planning observations and relative dark calibration.

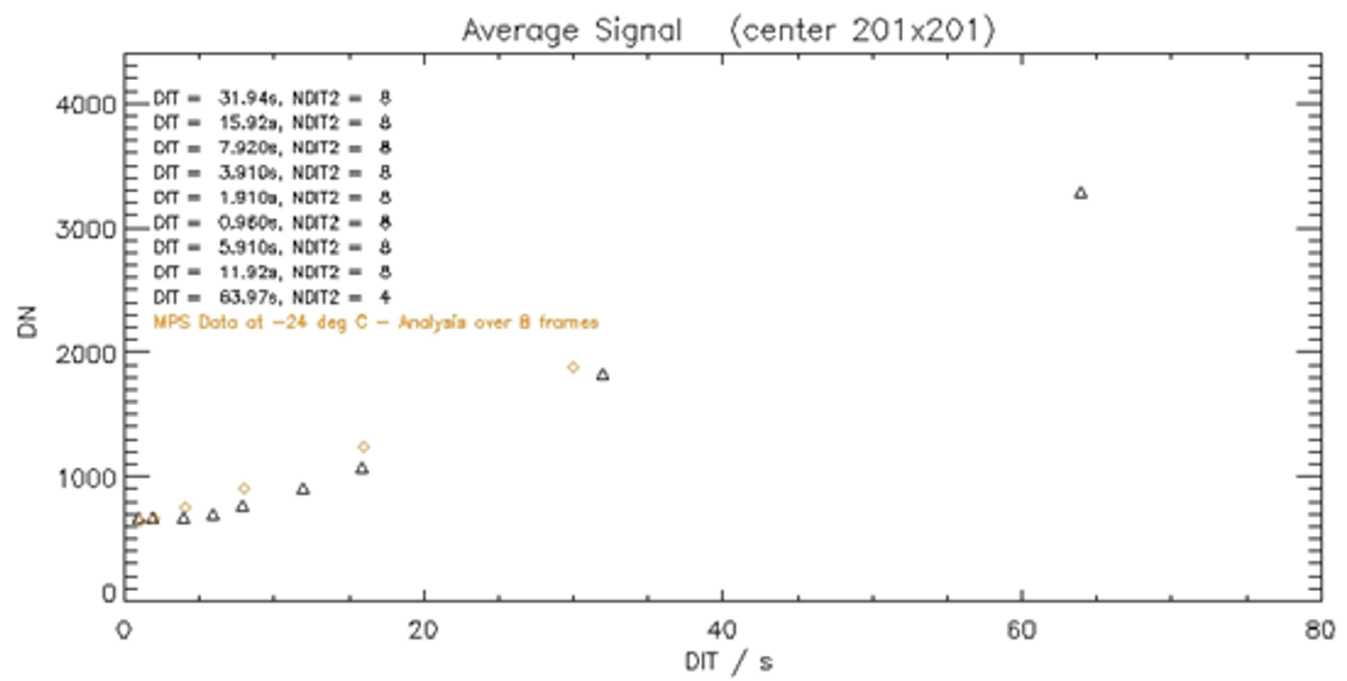

Figure 6. UV dark trend with DIT at $T=-26^{\circ} \mathrm{C}$, compared with on-ground test trend.

A temporary acquisition scheme has been devised. The image frames taken in the first 70-80s of each acquisition have a significantly lower average level due to the transient effect. For these reasons the following procedure is suggested:

- for each observational window dark frames shall be acquired with the same DIT used for science acquisitions, and possibly with the same NDIT1/NDIT2;

- a number of dark frames shall be acquired for each DIT in order to sample the full rising edge of the transient and the plateau

With this approach, it is possible to limit the effect of the transient. However, further investigations are planned to solve the problem, by studying the causes of this effect in the way the software operates the camera and the feasibility of implementing in the on board software a delay in the acquisition sequence.

\subsection{Metis Cap Ejection and First Light}

Metis cap was designed to protect the inner environment of Metis from molecular and particle contamination since instrument integration throughout the launch phase. Metis requirements on cleanliness are very demanding. Particle contamination must be minimized because in a coronagraph it is important to reduce all sources of stray light produced by the solar disk radiation that can overwhelm the several orders of magnitude $(>6)$ dimmer 


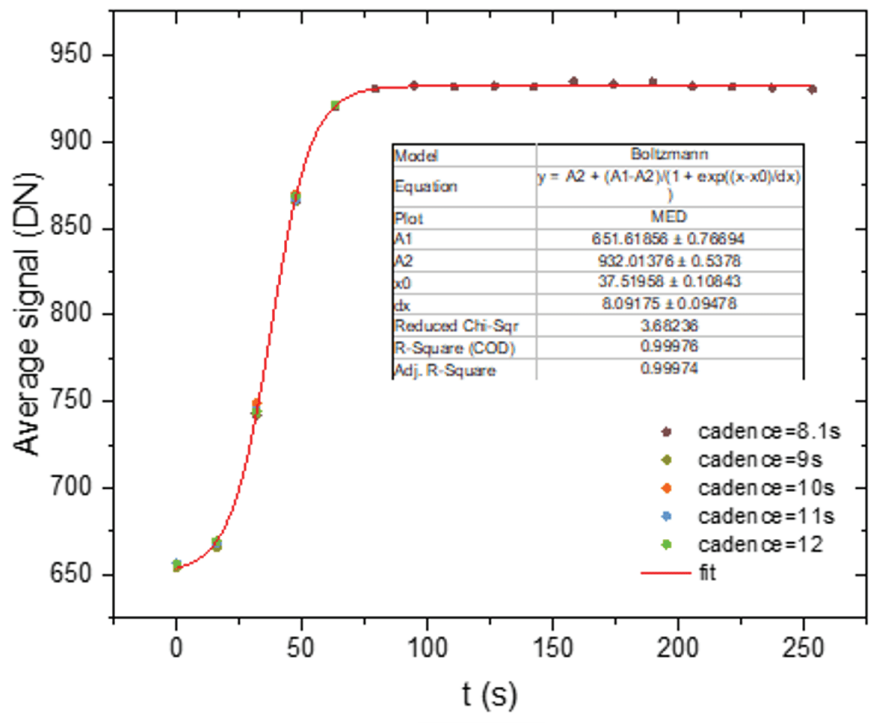

Figure 7. Data sets plotted on the same time scale (each series starts at $t=0 \mathrm{~s}$ ).

coronal radiation. Any dust grain larger than $50 \mu \mathrm{m}$ placed in the radiation path can scatter radiation that swamps the coronal brightness. Molecular contamination deposited on the telescope mirror reduces the efficiency in the EUV. Metis cap was procured by ESA and consisted of a one shot ejectable cap closing the IEO aperture. The ejection was mechanically triggered by the opening the heat shield door for the first time. The cap was successfully ejected during IT-3 after about one month from launch to allow a full internal outgassing and the first VL images were acquired: two polarized brightness $(\mathrm{pB})$ images and a sequence of 5s exposure imges at fixed polarization while the heat shield door was closing. Fig. 8 shows the first visible-light image, background subtracted, acquired by Metis. All acquired images show clear signatures of stray-light contamination, with bright patterns located at the top-left and bottom-right corners of the detector frame. In these regions the saturation level is reached.

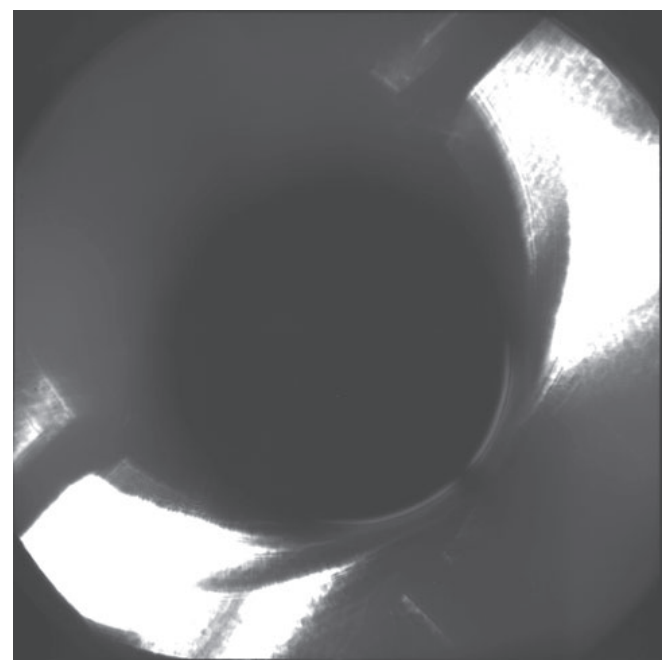

Figure 8. VL first light evendences a misalignment of the occultation system. 
During the following commissioning activity, IT-4, the UVD was commissioned. The high-voltage was safely ramped up step by step to the operative value of $814 \mathrm{~V}$ for the MCP and $5000 \mathrm{~V}$ for the phosphor screen. Fig.9 shows the first UV image, background subtracted, acquired by Metis. The stray-light contamination is visible in the lower left quadrant, but to a much lesser extent than that affecting the visible-light channel, as expected due to the dependence of diffraction on the wavelength and the lower contrast between disk and corona, thus Lyman- $\alpha$ emission from the coronal streamer belt has been clearly detected by the instrument.

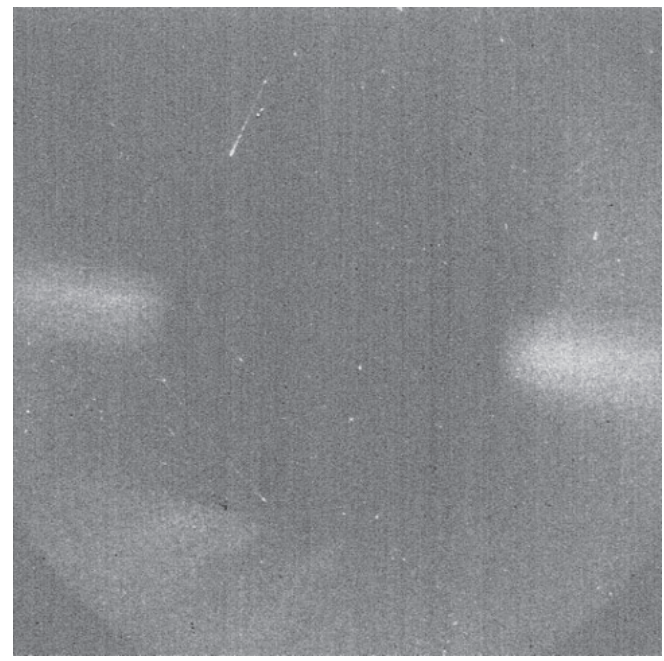

Figure 9. UV first light confirms a misalignment of the occultation system. Stray light pattern is visible in the third quadrant of the image.

After IT-4 all instrument sub-systems have been commissioned.

\subsection{Internal Occulter Coarse Optimization and First Coronal Brightness Images}

The first images in both channels show a slight misalignment of the occulting system. The occulting system consists of four components: the IEO, the disk light rejection mirror (M0), the internal occulter, IO, and the Lyot Stop. The IO is a circular stop that occults the light diffracted by the edge of the IEO, and the Lyot stop that blocks the light from the edge of M0, both imaged by the primary mirror. The Lyot stop, a circular occulter mounted in the back of M0, is less critical because it stops a secondary diffraction source. The on-ground alignment verification showed a similar misalignment that was corrected. The second misalignment could be due to an impulse received by the IO during launch. These event confirm the importance of the presence of an IO mechanism to compensate for accidental misalignments during the integration and launch phases, especially for the most stray light sensitive visible channel. The IO mechanism consists of two stepper motor actuators placed along crossed directions that support the IO. Each step actuation translates the IO by about $6 \mu \mathrm{m}$. The coarse alignment was carried out by performing a 10 step wide grid of positions for the two actuators and taking VL images for each position. The amount of IOM shift was formulated on the basis of the comparison of the stray light features between IT-3 and on-ground calibration images before IOM optimization. Both a qualitative and a quantitative comparison were performed, despite some physical differences between the two configurations (simulated heliocentric distance in the laboratory $0.5 \mathrm{AU}$, distance in IT-3, $0.95 \mathrm{AU}$. In the laboratory, the source was a stabilized solar simulator with no corona; in IT-3, the actual Sun). A qualitative comparison between a laboratory image before IOM optimization, an IT-3 image and a Zemax OpticStudio (C) simulation with decentered IOM is shown in Fig.10.

The quantitative analysis is performed by taking a selected number of regions of interest in the image, drawn around the brightest stray light features. All analyses were in agreement: there was a slight misalignment along one direction (about 30 steps), and a negligible one along the perpendicular direction. The fine optimization of the IO foresees a grid of 3 step intervals. It was planned within the same activity slot IT-6. Due to a loss of 

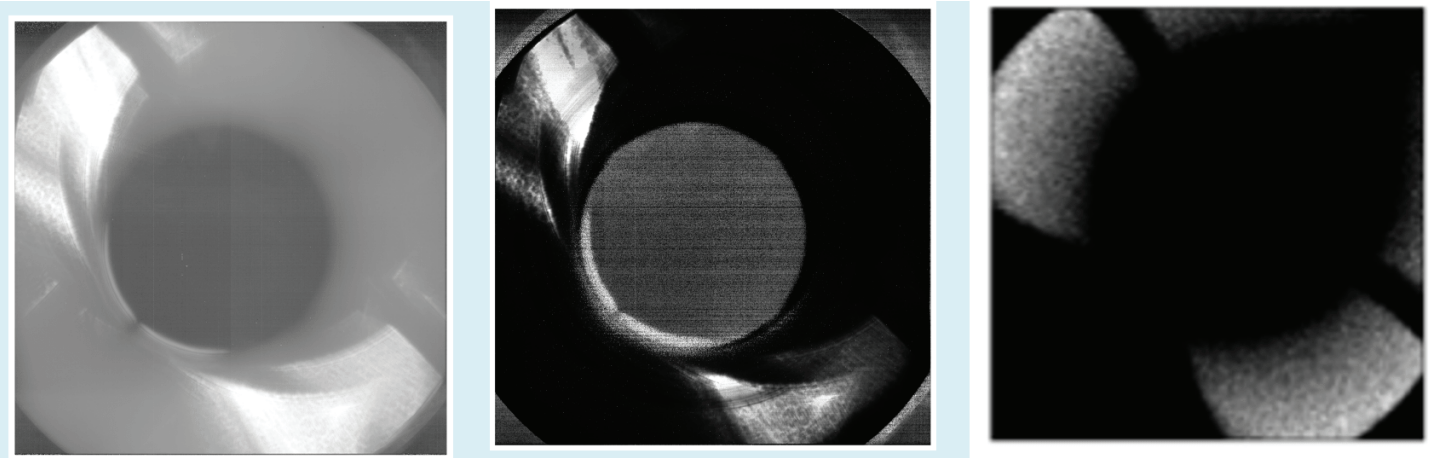

Figure 10. Comparison between IT-3 (left), on-ground calibration before IOM optimization (middle) and a Zemax OpticStudio $^{(}$simulation with off-centered IOM (right).

contact with the spacecraft by the deep space network antenna, the activity was resumed without HSD opening and it had to be replanned within the Cruise Phase (February 2021). The fine optimization is particularly important when the spacecraft will approach the minimum perihelion and the disk overocculting will be reduced to minimum.

\subsection{Stray Light Characterization}

Metis stray light is caused by three main factors:

- Dust on the IEO edge or solid fragment passing in front of the IEO. This is either a not removable (dust) or transient contribution, and won't be discussed any longer.

- Metis pointing off the solar disk center.

- IO not aligned with the IEO image.

A combination of the last two cases is in any case possible.

Given the large distance from the Sun, always above $0.5 \mathrm{AU}$, the Sun is very overocculted and we have stray light levels which are well below the required stray light rejection of $10^{-9}$ in mean disk brightness units. We have to wait till the first perihelion of 2022 for an assessment of Metis stray light performance.

Two schemes have been used during commissioning. The first one involves a roll of the spacecraft around a sun-centered axis; the second one involves spacecraft off-pointings to bring the limb of the Sun close to the edge of the Sun disk rejection mirror.

In order to disentangle the stray light contributions on the basis of their source, a $180^{\circ}$ roll maneuver of the spacecraft is performed. The procedure to analyze the set of data is sketched in Fig.11.

Two acquisitions are performed, Acq1 in the nominal spacecraft position and Acq2 with a spacecraft roll of 180 degrees. Then, an image rotation of 180 degrees is performed on Acq2 and the rotated Acq 2 is subtracted from Acq1. If the spacecraft is Sun center pointed, then the resulting image will have two distributions of negative and positive pixels exactly symmetric with respect to the frame center. If the spacecraft is not pointed, then the symmetry will be broken (as in the bottom panel of Fig.11). In order to understand which of the three misalignment cases was better fitting to the Metis first light (acquired in IT-3), a spacecraft 180 degrees roll was performed in IT-6A, before the IO optimization, and images were acquired before and after it.

The difference image (Fig.12) shows an excellent symmetry between the positive (white) and negative (black) pixels distributions with respect to the frame center. It is a result that demonstrates that the main cause for the IT-3 and IT-4.3 stray light dominated images is the misalignment between IEO and IO and is totally internal to Metis. 

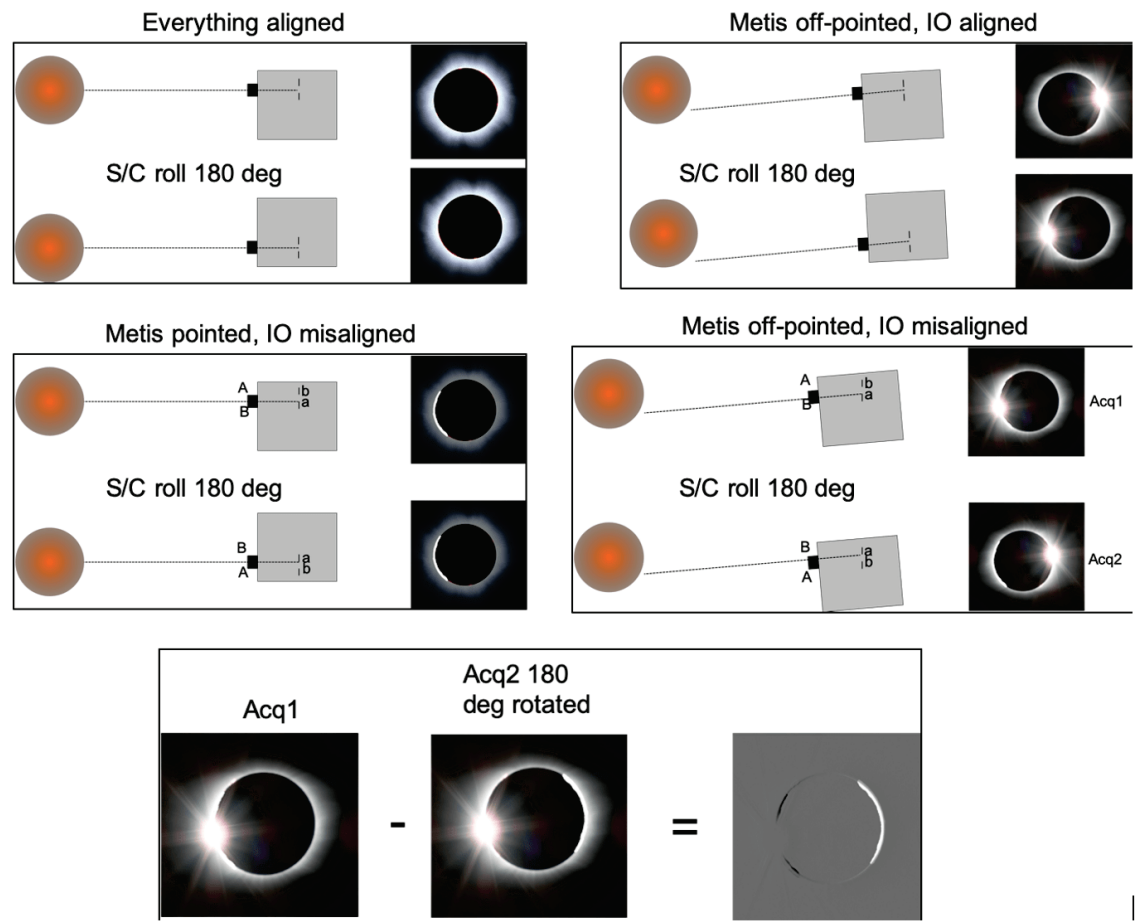

Figure 11. Procedure to disentangle stray light contributions on the basis of their source.
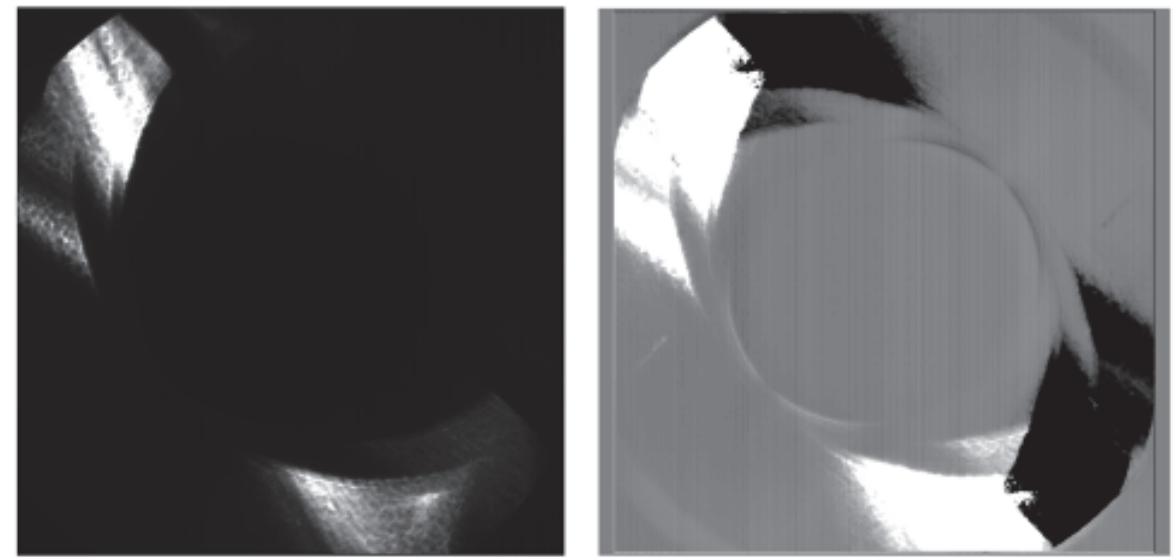

Figure 12. Left: IT-6A Acq1 (before spacecraft roll). Right: difference (Acq1)-(Acq2 180 degrees rotated).

An additional test will be performed during the cruise phase.

The second scheme has been applied during the IT-7 activity. The spacecraft performs slews on both sides of the disk center across the North-South and the East-West directions with constant steps of $0.1^{\circ}$. The acquired images, both total brightness $(\mathrm{tB})$ and $\mathrm{pB}$ images, are de-vignetted with the in-flight vignetting function, shown in Fig.13, and shifted in order to overlap the same coronal structures. Finally a running difference over the subsequent slews is taken. The stray light was evaluated by averaging the signal in sectors, close to the inner portion of the FoV (Fig.14) and display the value as a function of the slew, as shown in Fig.15 for both tB and $\mathrm{pB}$. The result in $\mathrm{pB}$ is significantly lower with respect to the analogous plot obtained with pB single frames, 


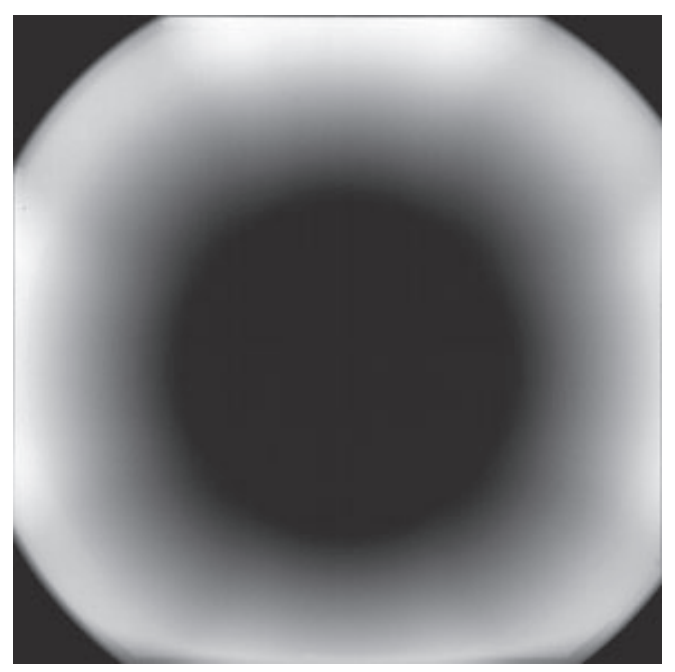

Figure 13. VL in-flight vignetting function. ${ }^{6}$

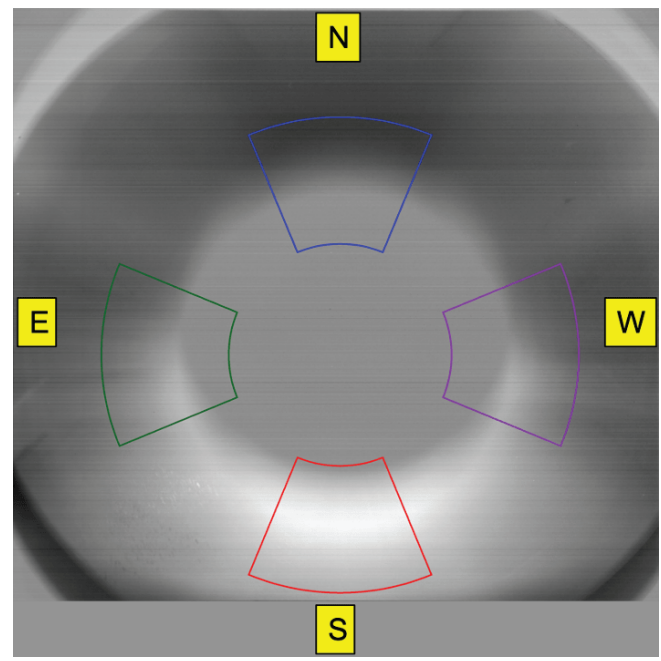

Figure 14. Difference image. Stray light was evaluated by taking averages in the color sectors drawn on difference images.

which is a confirmation that stray light is not polarized or has a very small polarized component.

\subsection{Polarimeter Characterization}

Metis visible light channel measures the linear polarization of the solar corona in order to isolate the Thompson scattered component of the solar corona and derive the electron density. Metis polarimeter ${ }^{2}$ package consists of a quarter wave retarder plate, a liquid crystal variable retarder (LCVR) plate and a linear polarizer. There are no moving parts and the polarization is modulated by varying the voltage across the double cell of the $\operatorname{LCVR}^{7,8}$

One of the specific goals of the IT-7 campaign was the extensive test of the polarimeter functionality, already tested during the on ground campaign. ${ }^{9}$ During the roll maneuver, the Metis polarimeter acquired several sets of images of the K-corona polarized brightness $(\mathrm{pB})$ for each of the 8 roll positions going through a full roll in steps of $45^{\circ}$. The goal is to provide a calibration of the LCVR and to check the polarized flat-field. 

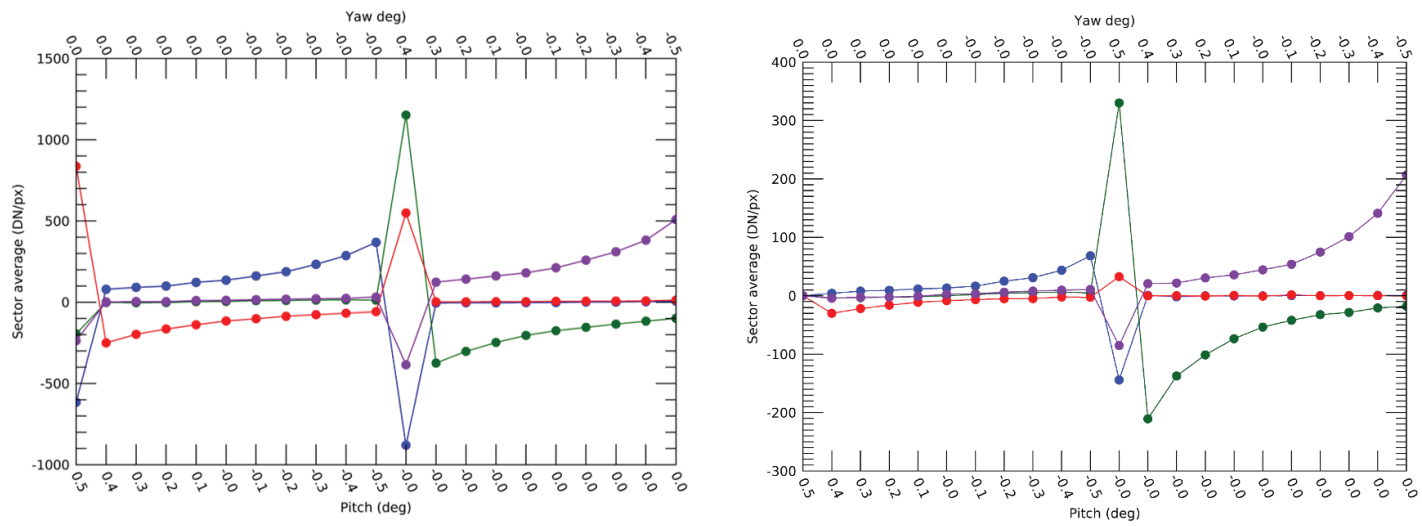

Figure 15. Running differences on tB (left) and pB (right) images. The average taken in sectors described in Fig.14, with the same color code.

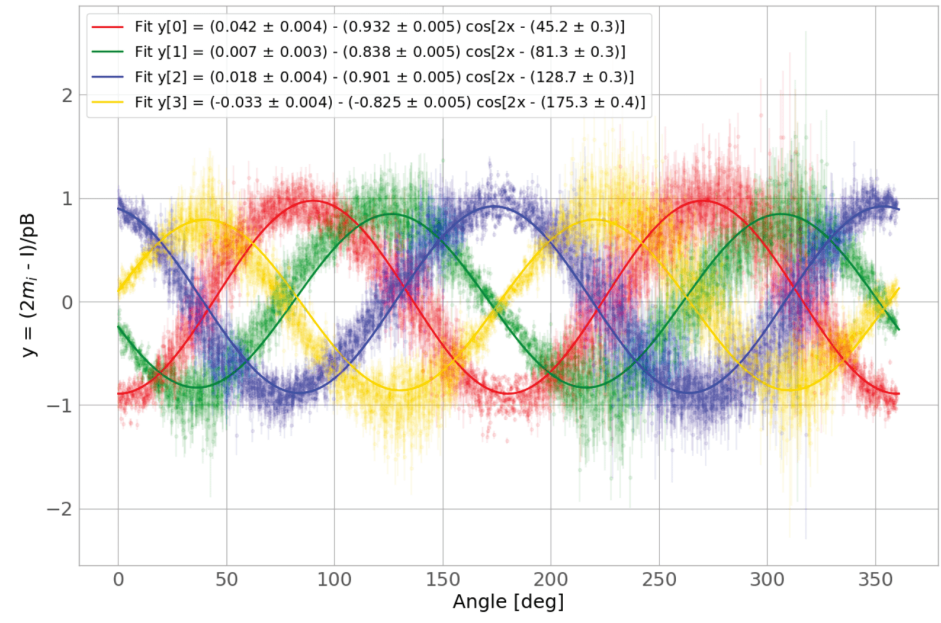

Figure 16. Plot of the 4 regressions to perform the in-flight calibration of the LCVR retardances. The data come from a quadruplet acquired during the first roll position of the IT-7 campaign for a fixed heliocentric height.

The in-flight calibration of the LCVR retardances is obtained considering the geometric properties of the light polarization in the solar corona (i.e. linear polarization tangent to the solar limb). Considering 4 corona images at different linear polarizations, $m_{i}$ and the Stokes vector in the reference system of the image, for a point $\mathrm{P}$ with coordinates $(r, \alpha)$, we can rewrite the recorded signal for each sensor element such as:

$$
\frac{\left(2 m_{i}-I\right)}{p B}=-\cos \left(2 \alpha-\delta_{i}\right)
$$

where $\delta_{i}$ are the retardance values that can be estimated through the 3-parameters regression:

$$
y_{i}=P_{0}-P_{1} \cos \left(2 \alpha-P_{2}\right)
$$

where $y_{i}=\frac{\left(2 m_{i}-I\right)}{p B}, P_{0}$ is the offset of the fitting curve, $P_{1}$ is the modulation amplitude and $P_{2}=\delta_{i}$, using the same region in the solar corona. tangent to the limb, therefore, the $\mathrm{S} / \mathrm{C}$ rotation gives the same result as the rotation of the polarizer. We therefore expect that the recorded intensities follow the Malus law (Fig.16). 
From the average of the values at different heliocentric heights, we obtain: $\left(45.0^{\circ} \pm 0.1^{\circ}, 81.4^{\circ} \pm 0.1^{\circ}, 128.7^{\circ} \pm\right.$ $0.1^{\circ}, 175.4^{\circ} \pm 0.1^{\circ}$ ) that are consistent with the expected ones.

A second test using the spacecraft roll is to check the polarized flat-field: the $\mathrm{pB}$ image of the same coronal structure was measured in different locations of the detector focal plane. The comparison of the $\mathrm{pB}$ images will give indications of the differences in the polarimetric response from different locations across the polarimeter and the detector. Quantitatively, we can plot the value of the $\mathrm{pB} / \mathrm{I}$ variation for each roll in a fixed region on the frame (for example along a streamer). Considering this region and following it the $\mathrm{S} / \mathrm{C}$ rotation during the 8 different rolls (Fig.17) maintaining the fixed distance from the Sun center (found by astrometry), we expect to find almost the same values of $\mathrm{pB} / \mathrm{I}$.
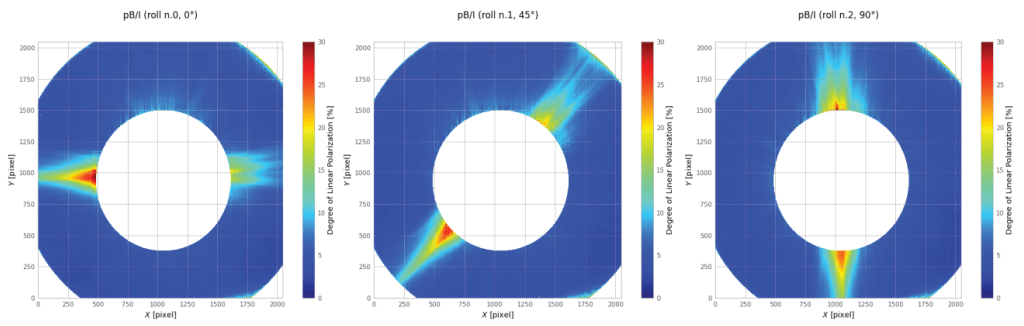

pBA (rol $1.5 .225^{\circ}$
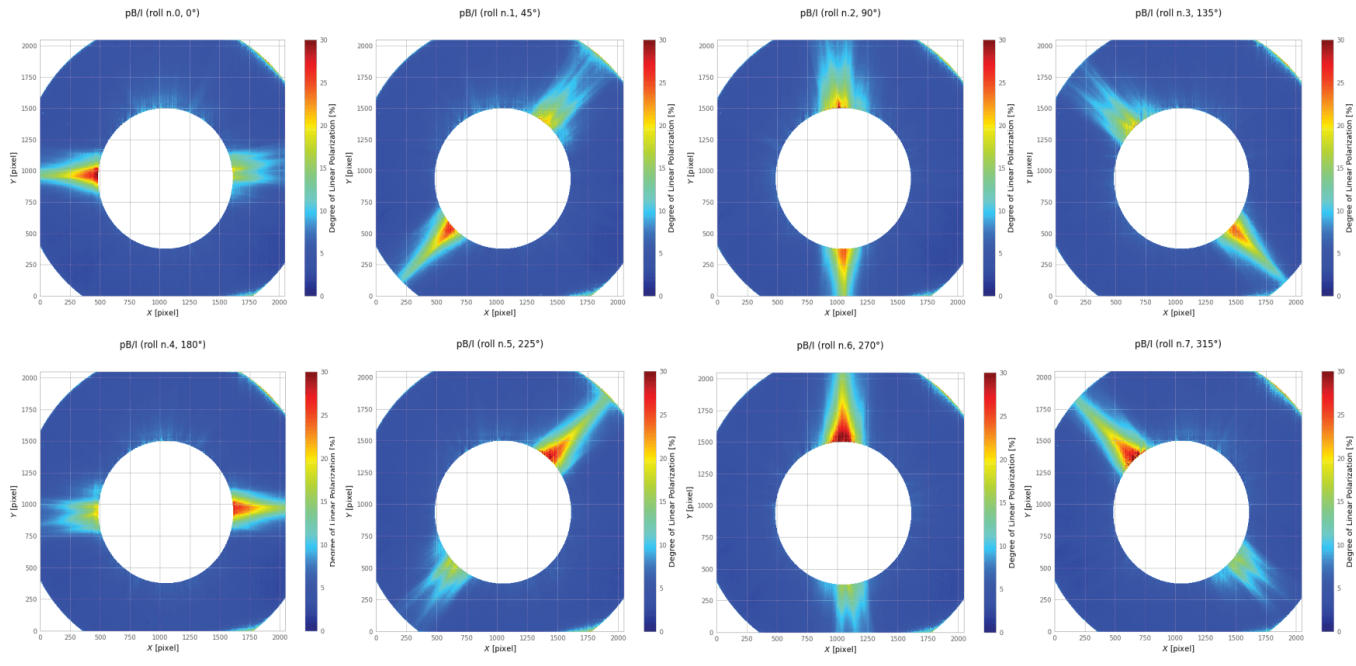

(roll $n .7,315^{\circ}$

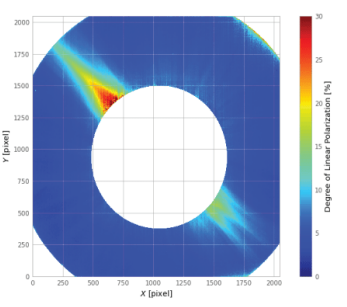

Figure 17. Degree of linear polarization $(\mathrm{pB} / \mathrm{I})$ of the K-corona for the 8 different $\mathrm{S} / \mathrm{C}$ roll positions.

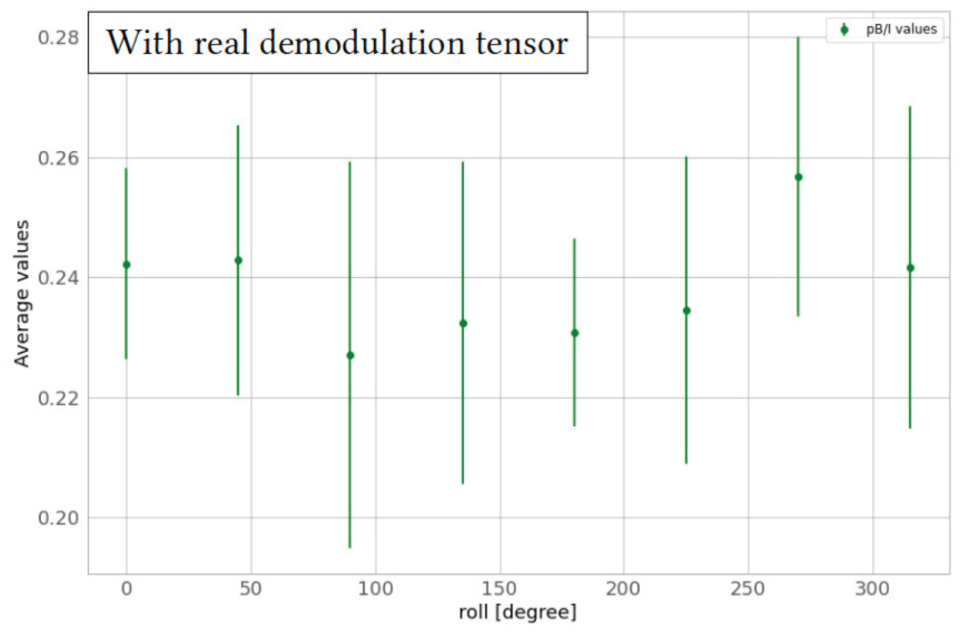

Figure 18. pB/I average over the pixels inside a selected regions (for each roll) using the demodulation tensor obtained during the ground calibration. The bars represent the dispersion inside the considered area.

Fig.18 shows the result for a selected region at $\sim 4$ solar radii inside the streamer. The pB/I values are consistent with the expected values (i.e. the $\mathrm{pB} / \mathrm{I}$ value at the same heliocentric height is almost constant for 
different roll). The average value is: $p B / I=0.25 \pm 0.01$ with an averaged percentage error $\sim 3 \%$. A more complete description of this tests is given in the paper of Liberatore et al., in this issue. ${ }^{6}$

\subsection{Cosmic Ray Algorithm, Compression and Radialization Test}

In order to minimize the data to be downloaded, Metis has an on-board compression algorithm able to perform both lossless or lossy compression. The algorithm, developed by the Politecnico of Turin, improved the ESA predictor CCSDS-123 to include the possibility for the lossy compression and optimized it to the Metis case. The algorithm works on the data stream coming out from previous tasks that select the region of interest (masking) and the resolution of the frame (binning). The algorithm takes advantage of the expected roughly radial symmetry of the Metis images to apply a different compression in distinct annular regions of the FOV. The so called 'radialization' procedure allows the user to select up to 3 different annular belts where a different compression quality can be set. During NECP most of the images have been acquired with neither binning nor masking and using a uniform lossless compression. In this condition the compression ratio achieved, see fig. 19, is roughly 2 slightly varying with the detector integration time due to the different dynamic range of the acquired image (the wider the signal to be compressed the lower the efficiency of the compression).
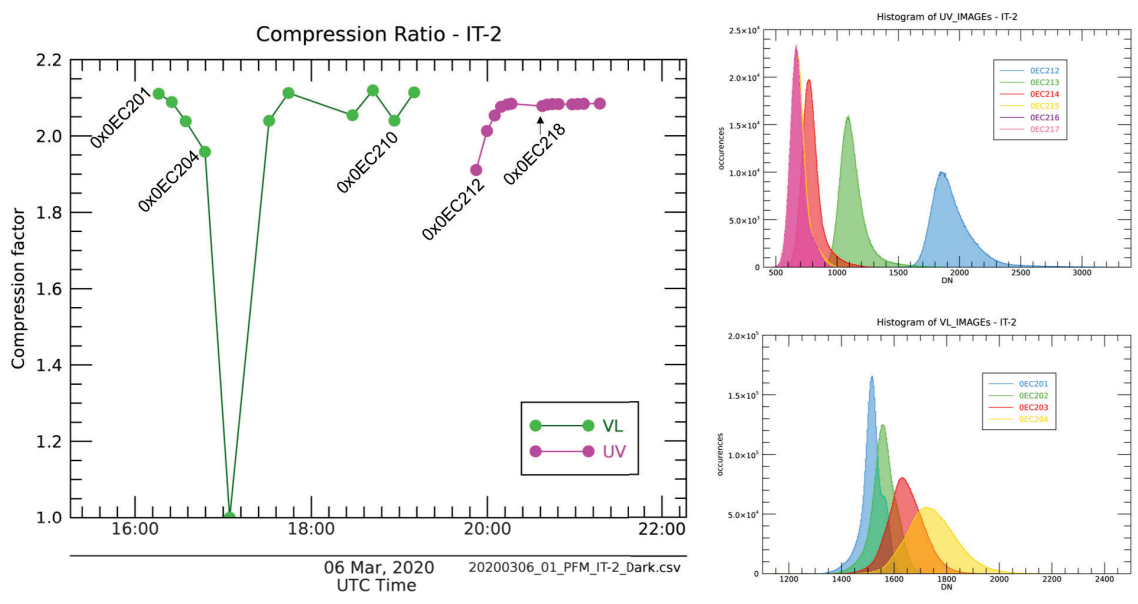

Figure 19. Compression Ratio achieved during IT-2 campaign (left panel) for the UV and VL Metis detector. Note that the fifth VL image had the compression erroneously disabled and therefore provided a $\mathrm{CR}=1$. On the right the histogram of the pixels values of some representative IT-2 images are shown. As expected the images with more spread histogram (wider dynamic range) provide a higher compression ratio.

Some dedicated tests have been carried out to verify more complex compression configurations. In particular during IT-5 and IT-7 some functional tests have been run using the uniform binning and the masking combined with radialization using the variable lossy compression. In case a $2 \times 2$ uniform binning is applied, the lossless compression efficiency improves of a $\sim 50 \%$ factor achieving an overall compression ratio of $\sim 12$.

The most complex configuration foresaw a combination of masking and variable lossless and lossy compression. The loss of information introduced by the compression algorithm can be tuned providing a parameter, $\Delta \mathrm{Q}$, that measures the possible error on the signal of a retrieved pixel with respect to its original value. The greater the value of $\Delta \mathrm{Q}$, the higher the loss of information (and therefore the higher the compression ratio achieved). The commanded compression foresaw a masking of the central part of the image (roughly behind the Metis occulter), a wide annular region lossless compressed and the outer regions lossy compressed with a $\Delta \mathrm{Q}$ of 5 and $10 \mathrm{DN}$ respectively as sketched in figure 20. The retrieved images show no relevant visible artifacts providing an overall compression ratio greater than 3. This kind of acquisitions have been taken only as functional check and it does not represent the configuration for the nominal acquisition, anyway it represents a viable way to improve the compression efficiency with a minimal loss of quality and maintaining the higher spatial resolution. 


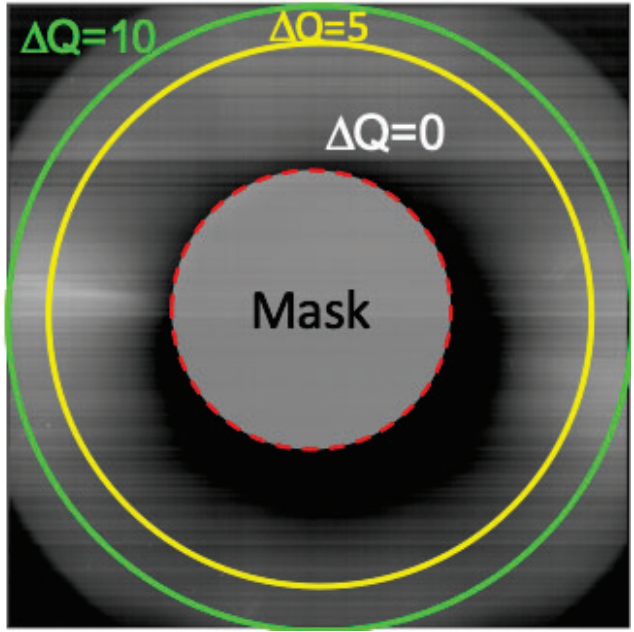

Figure 20. Configuration adopted for the most complex setting tested for the compression algorithm.

During IT-5 was tested also the CR_SEP Algorithm, a routine that aims at removing the tracks left by the cosmic rays and the solar energetic particles hitting the Metis detectors. The algorithm works on the single acquired frames before the on board averaging and compares the signals registered on the consecutive frames. A proper threshold allows to identify the possible tracks left by an energetic event in one of the comparing frames. Some observations have been dedicated to test this algorithm. In particular in 2 sessions a series of 10 images with no averaging on-board and with the CR_SEP algorithm disabled were acquired. The following acquisitions have been performed averaging 10 frames on-board and enabling the CR_SEP algorithm. The same algorithm has been implemented on ground and applied to the frames not corrected on-board (first session). The results have been compared with that obtained on-board. While the VLDA provides a result that is quite similar, the UVDA shows different performance. This unexpected behavior is probably linked to a known effect that caused an enhancement of the bias level for the first frames acquired by the UVDA and that is still under investigation. Further tests have been already planned for the next observing windows.

\subsection{Pointing assessment}

Star observations within Metis FOV are very important to characterize and calibrate the instrument. In the VL channel, stars are commonly seen in the FOV and are often bright enough to stand out above the solar corona. Stars are also visible in the UV channel: they are typically early-type bright stars (type O and B) which emit conspicuously in the Metis UV narrow bandpass. Passes of this type of stars within Metis FOV are traced and represent a target of opportunity for Metis calibration and for cross calibration with EUI and SPICE.

Stars observations have multiple scopes:

- determine the center of the solar disk in the image;

- probe the spatial resolution of the coronagraph (see Da Deppo et al., in this issue ${ }^{10}$ );

- determine the radiometric calibration (activity still in progress).

A coronagraph is designed to operate sun center pointed, but determining the instrument boresight and the position of the Sun center in the image is not straightforward, because the Sun is occulted. Fig.21 shows the relevant reference frames used in determining the instrument pointing, with all offsets and angles exaggerated for display purposes.

The reference frames shown in the figure are the following: 

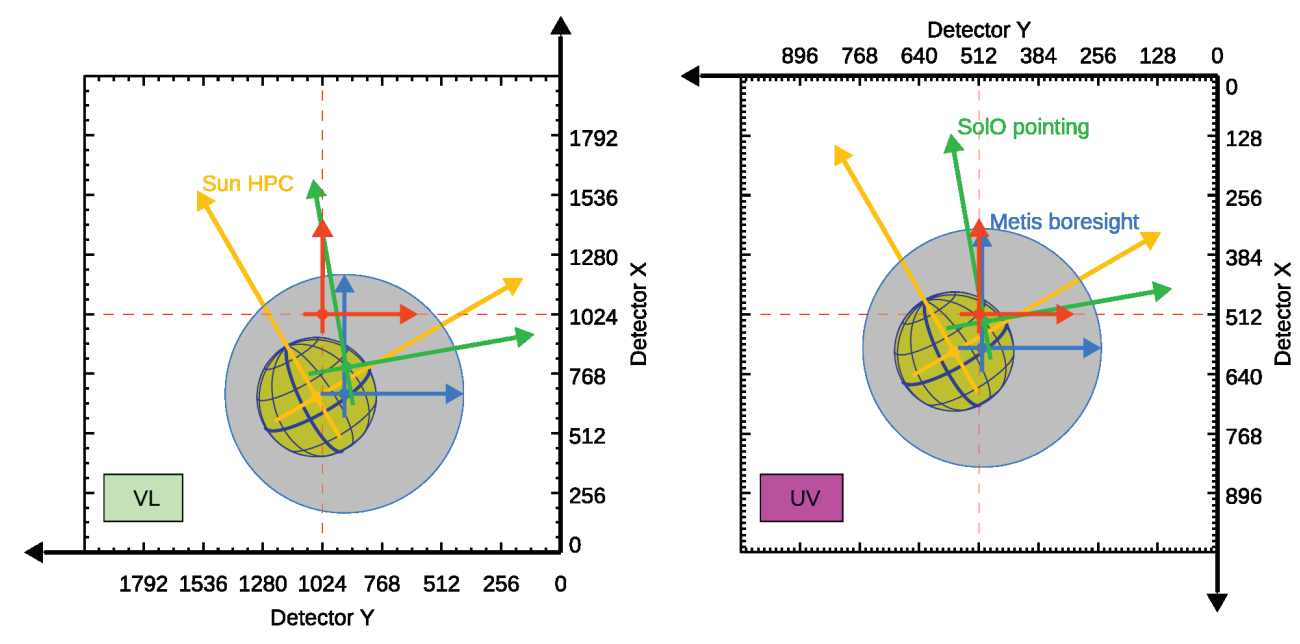

Figure 21. Metis UV and VL reference frames: detectors' reference frames (black and red), instrument reference frame (blue), spacecraft reference frame (green), helioprojective cartesian reference frame (HPC) (yellow). For completeness, a Stonyhurst heliographic grid is also shown. Relative displacement and rotations between the various reference frames are amplified for better display clarity.

- The detector reference frames for the two channels, $x_{\text {det }}$ and $y_{\text {det }}$, are shown in black, starting from the $(0,0)$ pixel, corresponding to the $(1,1)$ pixel in FITS convention.

- The Sun-oriented detector reference frames, $X_{d}$ and $Y_{d}$, in red, i.e. a reference frame on the detectors whose $\mathrm{X}$ axis points towards the solar West and the $\mathrm{Y}$ axis points to the solar North in the case spacecraft roll with respect to the solar North is zero.

- The instrument reference frame, in blue, whose axes are parallel to the detector axes, but the origin is at the instrument boresight, $\left(X_{b}, Y_{b}\right)$, which is defined as the center of the shadow of the Internal Occulter (IO). The shadow of the IO is shown as a grey disk.

- The spacecraft reference frame, in green, with the origin at $\left(X_{p}, Y_{p}\right)$; the $\mathrm{S} / \mathrm{C}$ pointing is normally obtained from the orbital and attitude parameters available at the time of the image acquisition.

- Finally, the solar HelioProjective Cartesian (HPC) reference frame, or solar reference frame, in yellow, with center at $\left(X_{s}, Y_{s}\right)$.

Both the rotation angle ("S/C roll", or $\rho$ ) of the $\mathrm{S} / \mathrm{C}$ reference frame with respect to the solar frame and the rotation angle (Metis residual angle, $\delta \rho$ ) with respect to the $\mathrm{S} / \mathrm{C}$ reference frame increase counterclockwise (thus, the representative roll angles shown in figure are both negative). The value $\delta \rho$ is due to the misalignment of the instrument mount with respect to the $\mathrm{S} / \mathrm{C}$ reference frame and therefore is nearly constant, while $\rho$ will in general depend with time, according to the variation of S/C attitude as determined by planning or science requirements, for instance.

The instrument boresight $\left(X_{b}, Y_{b}\right)$ is defined by the direction of the axis that connects the center of M0 with the center of IEO. Nominally, it should be coincident with the telescope optical axis. The best way to determine the boresight of the instrument is to assume that the IO is on axis to best perform its diffraction blocking function. Then, the boresight is assumed to be the center of the vignetting function. In-flight, the vignetting function cannot be determined, and the boresight is the center of the central occultation shadow, both in the VL and in the UV. 
Table 2. Metis boresight and Sun center position during commissioning

\begin{tabular}{|l|c|c|}
\hline Sun center position $\left(X_{s}, Y_{s}\right)$ & $\begin{array}{c}\text { VL } \\
(+25.6 \pm 0.6,-57.2 \pm 0.8) \text { pixels } \\
(+259 \pm 7,-580 \pm 9) \text { arcsecs }\end{array}$ & $\begin{array}{c}(+2.63 \pm 1,-4 \pm 1) \text { pixels } \\
(+54 \pm 38,-83 \pm 38) \text { arcsecs* }\end{array}$ \\
\hline Plate scale $\left(S_{X}, S_{Y}\right)$ & $10.138 \pm 0.004$ arcsecs/pixel & $20.40 \pm 0.06$ arcsecs/pixel* \\
\hline Residual angle $(\delta \rho)$ & $-0.08 \pm 0.03$ degrees & $+0.23 \pm 0.15$ degrees* \\
\hline Pre-launch & $(+9.5,-59.5)$ pixels & $(-8.5,-4.5)$ pixels \\
Metis boresight $\left(X_{b}, Y_{b}\right)$ & $(+96,-603)$ arcsecs & $(-173,-92)$ arcsecs \\
\hline In-flight & $(+23.5 \pm 1,-85.5 \pm 1)$ pixels & $(1.6 \pm 2.5,-18.1 \pm 2.5)$ pixels \\
Metis boresight $\left(X_{b}, Y_{b}\right)$ & $(+238 \pm 11,-867 \pm 11)$ arcsecs & $(+33 \pm 40,-370 \pm 401)$ arcsecs** \\
\hline
\end{tabular}

Notes:

* UVD sun center position, plate scale and residual angle are measured during the RSCW1 in June.

** The position of the boresight for the UVD has not been to date measured; the value provided

in the last row of the table above was derived assuming that the shift between pointing and boresight, in angular units, is the same on both detectors; the positions in pixels were then derived using the VLDA pointing from $\left(X_{s}, Y_{s}\right)[\mathrm{UV}]+\left(\left(X_{s}, Y_{s}\right)[\mathrm{VL}]-\left(X_{b}, Y_{b}\right)[\mathrm{VL}]\right)$; errors were estimated by propagating the errors.

The solar reference frame can be found using stars in the Metis FoV. Star observations identify the world coordinate system on the FoV and therefore also the center of the Sun $\left(X_{s}, Y_{s}\right)$.

Tab.2 provides the first measurements of the Sun center position and Metis boresight for both VL and UV images as measured during the IT-6B1 IT-6B1 commissioning session where the optimization of the IO position has been completed.

\section{CONCLUSIONS}

Metis commissioning phase was run from February 27 to June 8, 2020 during the coronavirus lockdown restrictions. The activity was declared successfully completed at the ESA Mission Commissioning Results Review at the end of June, a couple of weeks before the first images were released to public.

Some of Metis features have not yet been commissioned, because either it was not possible during commissioning, like the CME flag, or, as in the case of the on-board software, because they need to be debugged on ground. A new version of the flight software is scheduled to be uploaded at the end of the Cruise phase, in November 2021.

The following features need to be commissioned:

- UV Photon Counting Unit

- Spatial noise function

- Sun disk monitoring

- CME watch

- Inter Instrument Communication (IIC)

None of these features are blocking Metis nominal activity.

Despite the large effort put in the commissioning phase, Metis team is still in the learning curve for the usage of the instrument and all the activities planned during the cruise phase will provide additional valuable pieces of information to begin the mission nominal phase at full regime at the beginning of 2022. Science data have already been acquired, fully showing the instrument potentiality. The main goal of the cruise phase is to provide a complete in-flight calibration package that is necessary for the data publication. 


\section{ACKNOWLEDGMENTS}

The Metis programme is supported by the Italian Space Agency (ASI) under the contracts to the co-financing National Institute of Astrophysics (INAF): Accordi ASI-INAF N. I-043-10-0 and Addendum N. I-013-12-0/1, Accordo ASI-INAF N. 2018-30-HH.0 and under the contracts to the industrial partners: ASI-TASI N. I-037-11-0 and ASI-ATI N. 2013-057-I.0. The Metis team thanks Barbara Negri, Enrico Flamini, Marco Castronuovo of the Italian Space Agency and Roberto Della Ceca, Giuseppe Malaguti of the Istituto Nazionale di Astrofisica for their continuous support during the development of the coronagraph. A special thanks to Filippo Marliani of the European Space Agency for his dedication to the program and his high standard of excellence. The testing of Metis was performed by an industrial consortium constituted by OHB Italia S.p.A. (acting as Prime Contractor towards ASI), Thales Alenia Space Italia S.p.A. (Co-Prime Contractor with the specific responsibility of the instrument AIT). ALTEC has provided logistics and technical support for the INAF Optical Payload Systems. The primary and secondary mirrors were provided as Czech contribution to Metis; the mirror hardware development was possible thanks to the Czech PRODEX Programme. The UVDA assembly was provided as a German contribution to Metis, thanks to the financial support of DLR (grant 50 OT 1201). The VLDA assembly was provided under Contract 2013- 058-I.0 with the Italian Space Agency (ASI). Metis team thanks the MOC personnel at ESOC for the support and the patience, in particular, Sylvain Lodiot, Alex Minogiannis and Ana Mestre. Metis team thanks the former PI, Ester Antonucci, for leading the development of Metis until the final delivery to ESA.

\section{REFERENCES}

[1] Müller, D., St. Cyr, O. C., Zouganelis, I., Gilbert, H. R., Marsden, R., Nieves-Chinchilla, T., Antonucci, E., Auchère, F., Berghmans, D., Horbury, T. S., Howard, R. A., Krucker, S., Maksimovic, M., Owen, C. J., Rochus, P., Rodriguez-Pacheco, J., Romoli, M., Solanki, S. K., Bruno, R., Carlsson, M., Fludra, A., Harra, L., Hassler, D. M., Livi, S., Louarn, P., Peter, H., Schühle, U., Teriaca, L., del Toro Iniesta, J. C., WimmerSchweingruber, R. F., Marsch, E., Velli, M., De Groof, A., Walsh, A., and Williams, D., "The Solar Orbiter mission. Science overview," 642, A1 (Oct. 2020).

[2] Antonucci, E., Romoli, M., et al., "Metis: the Solar Orbiter visible light and ultraviolet coronal imager," 642, A10 (Oct. 2020).

[3] Fineschi, S., Naletto, G., Romoli, M., Da Deppo, V., Antonucci, E., Moses, D., Malvezzi, A. M., Nicolini, G., Spadaro, D., Teriaca, L., Andretta, V., Capobianco, G., Crescenzio, G., Focardi, M., Frassetto, F., Landini, F., Massone, G., Melich, R., Nicolosi, P., Pancrazzi, M., Pelizzo, M. G., Poletto, L., Schühle, U., Uslenghi, M., Vives, S., Solanki, S. K., Heinzel, P., Berlicki, A., Cesare, S., Morea, D., Mottini, S., Sandri, P., Alvarez-Herrero, A., and Castronuovo, M., "Optical design of the multi-wavelength imaging coronagraph Metis for the solar orbiter mission," Experimental Astronomy 49, 239-263 (May 2020).

[4] Schühle, U., Teriaca, L., Aznar Cuadrado, R., Heerlein, K., Uslenghi, M., and Werner, S., "The solar orbiter Metis and EUI intensified CMOS-APS detectors: concept, main characteristics, and performance," in [Space Telescopes and Instrumentation 2018: Ultraviolet to Gamma Ray], den Herder, J.-W. A., Nikzad, S., and Nakazawa, K., eds., Society of Photo-Optical Instrumentation Engineers (SPIE) Conference Series 10699, 1069934 (July 2018).

[5] Solanki, S. K., del Toro Iniesta, J. C., Woch, J., Gandorfer, A., Hirzberger, J., Alvarez-Herrero, A., Appourchaux, T., Martínez Pillet, V., et al., "The Polarimetric and Helioseismic Imager on Solar Orbiter," 642, A11 (Oct. 2020).

[6] Liberatore, A. et al., "In-flight calibration of Metis coronagraph on board of Solar Orbiter," in [International Conference on Space Optics; ICSO 2020], Society of Photo-Optical Instrumentation Engineers (SPIE) Conference Series, this issue (2021).

[7] Zangrilli, L., Fineschi, S., and Capobianco, G., "Calibration of the EKPol K-corona imaging polarimeter," in [Solar Physics and Space Weather Instrumentation III], Fineschi, S. and Fennelly, J. A., eds., Society of Photo-Optical Instrumentation Engineers (SPIE) Conference Series 7438, 74380W (Aug. 2009).

[8] Fineschi, S., Zangrilli, L., Rossi, G., Gori, L., Romoli, M., Corti, G., Capobianco, G., Antonucci, E., and Pace, E., "KPol: liquid crystal polarimeter for K-corona observations from the SCORE coronagraph," in [Solar Physics and Space Weather Instrumentation], Fineschi, S. and Viereck, R. A., eds., Society of Photo-Optical Instrumentation Engineers (SPIE) Conference Series 5901, 389-399 (Aug. 2005). 
[9] Casti, M., Fineschi, S., Capobianco, G., Romoli, M., Antonucci, E., Nicolini, G., Naletto, G., Nicolosi, P., Spadaro, D., Andretta, V., Castronuovo, M., Massone, G., Susino, R., Da Deppo, V., Frassetto, F., Landini, F., Pancrazzi, M., Teriaca, L., and Uslenghi, M., "Metis/Solar Orbiter polarimetric visible light channel calibration," in [International Conference on Space Optics; ICSO 2018], Society of Photo-Optical Instrumentation Engineers (SPIE) Conference Series 11180, 111803C (July 2019).

[10] Da Deppo, V. et al., "In-flight optical performance assessment for the Metis solar coronagraph," in [International Conference on Space Optics; ICSO 2020], Society of Photo-Optical Instrumentation Engineers (SPIE) Conference Series, this issue (2021). 